\title{
Initial Value Problem for a Coupled System of Katugampola-Type Fractional Differential Equations
}

\author{
Yacine Arioua \\ University of M'sila \\ Laboratory for Pure and Applied Mathematics \\ Bp 166 M'sila, 28000, Algeria
}

\begin{abstract}
The aim of this work is to study the initial value problem of a coupled system of nonlinear fractional differential equations with Katugampola derivative. Some new existence and uniqueness results of solutions for the given problems are obtained by using the Banach contraction principle, Schauder's and nonlinear alternative Leray-Schauder fixed point theorems. Several examples are presented to illustrate the usefulness of our main results.
\end{abstract}

AMS Subject Classifications: 34A08, 34A12.

Keywords: Fractional differential equation, initial value problem, coupled system, fixed point theorem, existence, uniqueness.

\section{Introduction}

Fractional calculus is a mathematical branch which investigates the properties of derivatives and integrals of non integer orders (also known as fractional derivatives and integrals, briefly differ-integrals). The interested readers in the subject should refer to the books (Miller and Ross 1993 [11], Samko et al. 1993 [14], Podlubny 1999 [12], Kilbas et al. 2006 [10], Diethelm 2010 [4]).

The differential equations of fractional order are generalizations of classical differential equations of integer order; they are increasingly used in such fields as fluid flow, control theory of dynamical systems, diffusive transport akin to diffusion, probability and statistics ... etc.

Received November 28, 2018; Accepted February 2, 2019

Communicated by Sandra Pinelas 
In recent years, some authors have investigated the existence and uniqueness of solutions for a coupled system of nonlinear fractional differential equations. For a small sample of such work, we refer the reader to the works [1-3, 7, 13, 15-17].

In [1], Bashir Ahmad and Sotiris K. Ntouyas, studied a three point boundary value problem for a coupled system of nonlinear fractional differential equations given by

$$
\left\{\begin{array}{l}
\mathcal{D}_{0^{+}}^{\alpha} u(t)=f\left(t, v(t), \mathcal{D}_{0^{+}}^{p} v(t)\right), t \in(0,1), \\
\mathcal{D}_{0^{+}}^{\beta} v(t)=g\left(t, u(t), \mathcal{D}_{0^{+}}^{q} u(t)\right), t \in(0,1), \\
u(0)=0, u(1)=\gamma u(\eta), v(0)=0, v(1)=\gamma v(\eta),
\end{array}\right.
$$

where $1<\alpha, \beta<2, p, q, \gamma>0,0<\eta<1$, $\mathcal{D}$ is the standard Riemann-Liouville fractional derivative and $f:[0,1] \times \mathbb{R} \times \mathbb{R} \rightarrow \mathbb{R}$ are given continuous functions.

In [17], Zhai and Jiang considered a new coupled system of fractional differential equations with integral boundary conditions

$$
\left\{\begin{array}{l}
\mathcal{D}^{\alpha} u(t)+f(t, v(t))=a, 0<t<1 \\
\mathcal{D}^{\beta} v(t)+g(t, u(t))=b, 0<t<1 \\
u(0)=0, u(1)=\int_{0}^{1} \phi(t) u(t) d t \\
v(0)=0, v(1)=\int_{0}^{1} \psi(t) v(t) d t
\end{array}\right.
$$

where $1<\alpha, \beta \leq 2, a, b$ are constants. $\mathcal{D}$ denotes the usual Riemann-Liouville fractional derivative. $f, g \in C([0,1] \times \mathbb{R} \times \mathbb{R}), \phi, \psi \in L^{1}[0,1]$.

In this paper, we discuss the existence and uniqueness of solutions for a coupled system of Katugampola type fractional differential equations

$$
\left\{\begin{array}{c}
{ }^{\rho} \mathcal{D}_{0^{+}}^{\alpha} u(t)=f\left(t, u(t), v(t),{ }^{\rho} \mathcal{D}_{0^{+}}^{\alpha} u(t)\right) \\
{ }^{\rho} \mathcal{D}_{0^{+}}^{\beta} v(t)=g\left(t, u(t), v(t),{ }^{\rho} \mathcal{D}_{0^{+}}^{\beta} v(t)\right)
\end{array}, t \in[0, T],\right.
$$

with the initial condition

$$
u(0)=0, v(0)=0,
$$

where $0<\alpha, \beta \leq 1, \rho>0$, and $T \leq(p c)^{\frac{1}{p c}}$ for any $1 \leq p \leq \infty, c>0$, is a finite positive constant. The symbol ${ }^{\rho} \mathcal{D}^{\delta}, \delta=\alpha, \beta$, is the Katugampola fractional derivative of fractional order $\delta$, and $f, g:[0, T] \times \mathbb{R}^{3} \rightarrow \mathbb{R}$ are continuous functions.

Based upon a contraction mapping principle, Schauder's and nonlinear alternative Leray-Schauder's fixed point theorems. We obtain three various results of the existence and uniqueness about the initial value problem (1.1)-(1.2).

Throughout this paper, we assume the following hypotheses

(H1) $f, g:[0, T] \times \mathbb{R}^{3} \rightarrow \mathbb{R}$ are continuous functions.

(H2) There exists the constants $\lambda_{i}, \mu_{i}(i=1.2 .3)$, such that

$$
|f(t, u, v, w)-f(t, \tilde{u}, \tilde{v}, \tilde{w})| \leq \lambda_{1}|u-\tilde{u}|+\lambda_{2}|v-\tilde{v}|+\lambda_{3}|w-\tilde{w}|,
$$




$$
|g(t, u, v, w)-g(t, \tilde{u}, \tilde{v}, \tilde{w})| \leq \mu_{1}|u-\tilde{u}|+\mu_{2}|v-\tilde{v}|+\mu_{3}|w-\tilde{w}|,
$$

for any $u, v, w, \tilde{u}, \tilde{v}, \tilde{w} \in \mathbb{R}$ and $t \in[0, T]$.

(H3) There exist three positive functions $a_{i}, b_{i}, c_{i} \in C[0, T],(i=1.2)$, such that

$$
\begin{aligned}
|f(t, u, v, w)| & \leq a_{1}(t)+b_{1}(t)|u|+c_{1}(t)|v|+d_{1}(t)|w|, \\
|g(t, u, v, w)| & \leq a_{2}(t)+b_{2}(t)|u|+c_{2}(t)|v|+d_{2}(t)|w|,
\end{aligned}
$$

for all $t \in[0, T]$ and $u, v, w \in \mathbb{R}$.

We denote

$$
M_{i}=\frac{a_{i}^{*}}{1-d_{i}^{*}}, N_{i}=\frac{b_{i}^{*}+c_{i}^{*}}{1-d_{i}^{*}}, \text { and } K_{i}=\frac{b_{i}^{*}}{1-d_{i}^{*}}, H_{i}=\frac{c_{i}^{*}}{1-d_{i}^{*}},(i=1.2),
$$

where

$$
a_{i}^{*}=\sup _{t \in[0, T]} a_{i}(t), b_{i}^{*}=\sup _{t \in[0, T]} b_{i}(t), c_{i}^{*}=\sup _{t \in[0, T]} c_{i}(t), d_{i}^{*}=\sup _{t \in[0, T]} d_{i}(t), \text { with } d_{i}^{*}<1 .
$$

\section{Preliminaries}

In this section we present the necessary definitions from fractional calculus theory. As in [10], consider the space $X_{c}^{p}[0, T],(c \in \mathbb{R}, 1 \leq p \leq \infty)$, of those complex-valued Lebesgue measurable functions $y$ on $[0, T]$, for which $\|y\|_{X_{c}^{p}}<\infty$, where the norm is defined by

$$
\|y\|_{X_{c}^{p}}=\left(\int_{0}^{T}\left|s^{c} y(s)\right|^{p} \frac{d s}{s}\right)^{\frac{1}{p}}<\infty,
$$

for $1 \leq p<\infty, c \in \mathbb{R}$. For the case $p=\infty$

$$
\|y\|_{X_{c}^{\infty}}=e s s \sup _{0 \leq t \leq T}\left[t^{c}|y(t)|\right],(c \in \mathbb{R}) .
$$

By $C[0, T]$, we denote the Banach space of all continuous functions from $[0, T]$ into $\mathbb{R}$ with the norm

$$
\|y\|_{\infty}=\sup _{0 \leq t \leq T}|y(t)| .
$$

Remark 2.1. Let $p, c, T \in \mathbb{R}_{+}^{*}$, be such that $p \geq 1, c>0$, and $T \leq(p c)^{\frac{1}{p c}}$. It is clear that $\forall y \in C[0, T]$

$$
\|y\|_{X_{c}^{p}}=\left(\int_{0}^{T}\left|s^{c} y(s)\right|^{p} \frac{d s}{s}\right)^{\frac{1}{p}} \leq\left(\|y\|_{\infty}^{p} \int_{0}^{T} s^{p c-1} d s\right)^{\frac{1}{p}}=\frac{T^{c}}{(p c)^{\frac{1}{p}}}\|y\|_{\infty},
$$

and

$$
\|y\|_{X_{c}^{\infty}}=\operatorname{ess} \sup _{0 \leq t \leq T}\left[t^{c}|y(t)|\right] \leq T^{c}\|y\|_{\infty},
$$

which implies that $C[0, T] \hookrightarrow X_{c}^{p}[0, T]$, and

$$
\|y\|_{X_{c}^{p}} \leq\|y\|_{\infty}, \text { for all } T \leq(p c)^{\frac{1}{p c}} .
$$


Definition 2.2 (See [10]). The left-sided Riemann-Liouville fractional integral of order $\alpha>0$ of a continuous function $y:[0, T] \rightarrow \mathbb{R}$, is given by

$$
{ }^{R L} \mathcal{I}_{0^{+}}^{\alpha} y(t)=\frac{1}{\Gamma(\alpha)} \int_{0}^{t}(t-s)^{\alpha-1} y(s) d s, t \in[0, T]
$$

Definition 2.3 (See [10]). The left-sided Riemann-Liouville fractional derivative of order $\alpha>0$ of a continuous function $y:[0, T] \rightarrow \mathbb{R}$, is given by

$$
{ }^{R L} \mathcal{D}_{0^{+}}^{\alpha} y(t)=\frac{1}{\Gamma(n-\alpha)}\left(\frac{d}{d t}\right)^{n} \int_{0}^{t}(t-s)^{n-\alpha-1} y(s) d s, t \in[0, T], n=[\alpha]+1 .
$$

A recent generalization, introduced by Udita Katugampola (2011) [8], generalizes the Riemann-Liouville fractional integral and the Hadamard fractional integral (see [10]). The integral is now known as the Katugampola fractional integral, it is given in the following definition.

Definition 2.4 (See [8]). The Katugampola fractional integrals of order $\alpha>0$ of a function $y \in X_{c}^{p}[0, T]$, is defined by

$$
{ }^{\rho} \mathcal{I}_{0^{+}}^{\alpha} y(t)=\frac{\rho^{1-\alpha}}{\Gamma(\alpha)} \int_{0}^{t} \frac{s^{\rho-1} y(s)}{\left(t^{\rho}-s^{\rho}\right)^{1-\alpha}} d s, t \in[0, T]
$$

for $\rho>0$. These integrals are called left-sided integrals.

Similarly we can define right-sided integrals (See [8-10]).

Definition 2.5 (See [9]). The generalized fractional derivatives of order $\alpha>0$, corresponding to the Katugampola fractional integrals (2.1) defined for any $t \in[0, T]$, by

$$
\left.{ }^{\rho} \mathcal{D}_{0^{+}}^{\alpha} y(t)=\left(t^{1-\rho} \frac{d}{d t}\right)^{n}{ }^{(} \mathcal{I}_{0^{+}}^{n-\alpha} y\right)(t)=\frac{\rho^{\alpha-n+1}}{\Gamma(n-\alpha)}\left(t^{1-\rho} \frac{d}{d t}\right)^{n} \int_{0}^{t} \frac{s^{\rho-1} y(s)}{\left(t^{\rho}-s^{\rho}\right)^{\alpha-n+1}} d s,
$$

where $n=[\alpha]+1$, and $\rho>0$, provided the integrals exist.

Remark 2.6 (See [8,9]). As a basic example, we quote for $\alpha, \rho>0$, and $\mu>-\rho$

$$
{ }^{\rho} \mathcal{D}_{0^{+}}^{\alpha} t^{\mu}=\frac{\rho^{\alpha-1} \Gamma\left(1+\frac{\mu}{\rho}\right)}{\Gamma\left(1-\alpha+\frac{\mu}{\rho}\right)} t^{\mu-\alpha \rho} .
$$

Giving in particular

$$
{ }^{\rho} \mathcal{D}_{0^{+}}^{\alpha} t^{\rho(\alpha-m)}=0, \text { for each } m=1,2, \ldots, n .
$$


Theorem 2.7 (See $[8,9]$ ). Let $\alpha, \rho, c \in \mathbb{R}$, be such that $\alpha, \rho>0$. Then for any $f, g \in$ $X_{c}^{p}[0, T]$, where $1 \leq p \leq \infty$, we have

- Inverse property:

$$
{ }^{\rho} \mathcal{D}_{0^{+}}^{\alpha}{ }^{\rho} \mathcal{I}_{0^{+}}^{\alpha} f(t)=f(t), \text { for all } \alpha \in(0,1) .
$$

- Linearity property: for all $\alpha \in(0,1)$, we have

$$
\left\{\begin{array}{l}
{ }^{\rho} \mathcal{D}_{0^{+}}^{\alpha}(f+g)(t)={ }^{\rho} \mathcal{D}_{0^{+}}^{\alpha} f(t)+{ }^{\rho} \mathcal{D}_{0^{+}}^{\alpha} g(t), \\
{ }^{\rho} \mathcal{I}_{0^{+}}^{\alpha}(f+g)(t)={ }^{\rho} \mathcal{I}_{0^{+}}^{\alpha} f(t)+{ }^{\rho} \mathcal{I}_{0^{+}}^{\alpha} g(t) .
\end{array}\right.
$$

Definition 2.8 (Equicontinuous). Let $E$ be a Banach space. Call a part $P$ in $C(E)$ equicontinuous if

$$
\forall \varepsilon>0, \exists \delta>0, \forall u, v \in E, \forall \mathcal{A} \in P,\|u-v\|<\delta \Rightarrow\|\mathcal{A}(u)-\mathcal{A}(v)\|<\varepsilon .
$$

Theorem 2.9 (Ascoli-Arzela). Let $E$ be a compact space. If $\mathcal{A}$ is an equicontinuous, bounded subset of $C(E)$, then $\mathcal{A}$ is relatively compact.

Definition 2.10 (Completely continuous). We say $\mathcal{A}: E \rightarrow E$, is completely continuous if for any bounded subset $P$ of $E$, the set $\mathcal{A}(P)$ is relatively compact.

Lemma 2.11 (Gronwall. See [6]). Let $u(t)$ and $g(t)$ be nonnegative, continuous functions on $0 \leq t \leq T$, for which the inequality

$$
u(t) \leq \mu+\int_{0}^{t} g(s) u(s) d s, 0 \leq t \leq T,
$$

holds, where $\mu$ is a nonnegative constant. Then

$$
u(t) \leq \mu \exp \left(\int_{0}^{t} g(s) d s\right), 0 \leq t \leq T
$$

Theorem 2.12 (Banach's fixed point. See [5]). Let $P$ be a non-empty closed subset of a Banach space $E$, then any contraction mapping $F$ of $P$ into itself has a unique fixed point.

Theorem 2.13 (Schauder's fixed point. See [5]). Let E be a Banach space, and $P$ be a closed, convex and non-empty subset of $E$. Let $F: P \rightarrow P$ be a continuous mapping such that $F(P)$ is a relatively compact subset of $E$. Then $F$ has at least one fixed point in $P$.

Theorem 2.14 (Nonlinear Alternative of Leray-Schauder type. See [5]). Let E be a Banach space, $P$ be a closed, convex subset of $E, U$ be an open subset of $P$ and $0 \in U$ : Suppose that $F: \bar{U} \rightarrow P$ is a continuous, compact (that is, $F(\bar{U})$, is a relatively compact subset of $P$ ) map. Then either,

(i) F has a fixed point in $\bar{U}$; or

(ii) There is a point $u \in \partial U$ and $\mu \in(0,1)$ with $u=\mu F(u)$. 


\section{Main Results}

Throughout the remaining of this paper $T, p$ and $c$ are real constants such that

$$
p \geq 1, c>0, \text { and } T \leq(p c)^{\frac{1}{p c}} .
$$

In what follows, we present some significant lemmas to show the main theorems.

Lemma 3.1. Let $\alpha, \rho>0$. If $u \in C([0, T], \mathbb{R})$, then

(i) The fractional deferential equation ${ }^{\rho} \mathcal{D}_{0^{+}}^{\alpha} u(t)=0$, has a solutions

$u(t)=C_{1} t^{\rho(\alpha-1)}+C_{2} t^{\rho(\alpha-2)}+\ldots+C_{n} t^{\rho(\alpha-n)}$, where $C_{m} \in \mathbb{R}, m=1,2, \ldots, n$.

(ii) If ${ }^{\rho} \mathcal{D}_{0^{+}}^{\alpha} u \in C([0, T], \mathbb{R})$ and $0<\alpha \leq 1$, then

$$
{ }^{\rho} \mathcal{I}_{0^{+}}^{\alpha}{ }^{\rho} \mathcal{D}_{0^{+}}^{\alpha} u(t)=u(t)+C t^{\rho(\alpha-1)}, \text { for some constant } C \in \mathbb{R} .
$$

Proof. (i) Follows immediately from Remark 2.6.

(ii) If we apply the operator ${ }^{\rho} \mathcal{D}_{0^{+}}^{\alpha}$ to ${ }^{\rho} \mathcal{I}_{0^{+}}^{\alpha}{ }^{\rho} \mathcal{D}_{0^{+}}^{\alpha} u(t)-u(t)$, and use the properties (2.3), (2.4), we have

$$
\begin{aligned}
{ }^{\rho} \mathcal{D}_{0^{+}}^{\alpha}\left[{ }^{\rho} \mathcal{I}_{0^{+}}^{\alpha}{ }^{\rho} \mathcal{D}_{0^{+}}^{\alpha} u(t)-u(t)\right] & ={ }^{\rho} \mathcal{D}_{0^{+}}^{\alpha}{ }^{\rho} \mathcal{I}_{0^{+}}^{\alpha}{ }^{\rho} \mathcal{D}_{0^{+}}^{\alpha} u(t)-{ }^{\rho} \mathcal{D}_{0^{+}}^{\alpha} u(t) \\
& ={ }^{\rho} \mathcal{D}_{0^{+}}^{\alpha} u(t)-{ }^{\rho} \mathcal{D}_{0^{+}}^{\alpha} u(t)=0 .
\end{aligned}
$$

After the step (i) we deduce there exists $C \in \mathbb{R}$, such that

$$
{ }^{\rho} \mathcal{I}_{0^{+}}^{\alpha}{ }^{\rho} \mathcal{D}_{0^{+}}^{\alpha} u(t)-u(t)=C t^{\rho(\alpha-1)},
$$

After the step (i) we deduce there exists $C \in \mathbb{R}$, such that

$$
{ }^{\rho} \mathcal{I}_{0^{+}}^{\alpha}{ }^{\rho} \mathcal{D}_{0^{+}}^{\alpha} u(t)-u(t)=C t^{\rho(\alpha-1)},
$$

which implies the law of composition (3.1). The proof is complete.

Lemma 3.2. Let $0<\delta \leq 1$, and $\rho>0$. We give $y,{ }^{\rho} \mathcal{D}_{0^{+}}^{\delta} y \in C([0, T], \mathbb{R})$. Then the solution of problem

$$
\left\{\begin{array}{l}
{ }^{\rho} \mathcal{D}_{0^{+}}^{\delta} y(t)=f\left(t, y(t),{ }^{\rho} \mathcal{D}_{0^{+}}^{\delta} y(t)\right), t \in[0, T], \\
y(0)=0
\end{array}\right.
$$

is equivalent to the fractional integral equation

$$
y(t)=\int_{0}^{t} G_{\delta}(t, s) f\left(s, y(s),{ }^{\rho} \mathcal{D}_{0^{+}}^{\delta} y(s)\right) d s,
$$

where

$$
G_{\delta}(t, s)=\frac{\rho^{1-\delta}}{\Gamma(\delta)} s^{\rho-1}\left(t^{\rho}-s^{\rho}\right)^{\delta-1} .
$$


Proof. By applying ${ }^{\rho} \mathcal{I}_{0^{+}}^{\delta}$ to equation (3.2), we obtain

$$
{ }^{\rho} \mathcal{I}_{0^{+}}^{\delta}{ }^{\rho} \mathcal{D}_{0^{+}}^{\delta} y(t)={ }^{\rho} \mathcal{I}_{0^{+}}^{\delta} f\left(t, y(t),{ }^{\rho} \mathcal{D}_{0^{+}}^{\delta} y(t)\right) .
$$

From Lemma 3.1, we find easily

$$
{ }^{\rho} \mathcal{I}_{0^{+}}^{\delta}{ }^{\rho} \mathcal{D}_{0^{+}}^{\delta} y(t)=y(t)+C t^{\rho(\delta-1)},
$$

for some $C \in \mathbb{R}$. Then the fractional integral equation (3.2), gives

$$
y(t)={ }^{\rho} \mathcal{I}_{0^{+}}^{\delta} f\left(t, y(t),{ }^{\rho} \mathcal{D}_{0^{+}}^{\delta} y(t)\right)-C t^{\rho(\delta-1)} .
$$

In view of the initial condition $y(0)=0$, we get

$$
y(0)=0=-C \lim _{t \rightarrow 0^{+}} t^{\rho(\delta-1)} \Rightarrow C=0 .
$$

Therefore, the problem (3.2), is equivalent to

$$
y(t)=\int_{0}^{t} G_{\delta}(t, s) f\left(s, y(s),{ }^{\rho} \mathcal{D}_{0^{+}}^{\delta} y(s)\right) d s,
$$

where $G_{\delta}(t, s)$, which given by the equality (3.4). The proof is complete.

Based on the previous lemma, we will define the integral solution of the problem (1.1)-(1.2).

Lemma 3.3. Let $0<\alpha, \beta \leq 1$, and $\rho>0$. We give $u,{ }^{\rho} \mathcal{D}_{0^{+}}^{\alpha} u,{ }^{\rho} \mathcal{D}_{0^{+}}^{\beta} u \in C([0, T], \mathbb{R})$. Then the solution of problem (1.1)-(1.2) is equivalent to the coupled fractional integral equation

$$
\begin{aligned}
& u(t)=\int_{0}^{t} G_{\alpha}(t, s) f\left(s, u(s), v(s),{ }^{\rho} \mathcal{D}_{0^{+}}^{\alpha} u(s)\right) d s, \\
& v(t)=\int_{0}^{t} G_{\beta}(t, s) g\left(s, u(s), v(s),{ }^{\rho} \mathcal{D}_{0^{+}}^{\beta} v(s)\right) d s,
\end{aligned}
$$

with

$$
\begin{aligned}
G_{\alpha}(t, s) & =\frac{\rho^{1-\alpha}}{\Gamma(\alpha)} s^{\rho-1}\left(t^{\rho}-s^{\rho}\right)^{\alpha-1} \\
G_{\beta}(t, s) & =\frac{\rho^{1-\beta}}{\Gamma(\beta)} s^{\rho-1}\left(t^{\rho}-s^{\rho}\right)^{\beta-1} .
\end{aligned}
$$

Proof. In view of Lemma 3.2, for $\delta=\alpha$, and $\delta=\beta$, respectively. 
We will now prove a theorem about existence and uniqueness of solutions of initial value problem (1.1)-(1.2), which is based on Banach's fixed point theorem.

Let us introduce the space $E=X \times Y$, with the norm $\|(u, v)\|=\|u\|+\|v\|$, Obviously $(E,\|(u, v)\|)$, is a Banach space, where $X=\{u(t) / u(t) \in C([0, T], \mathbb{R})\}$, with the norm $\|u\|=\max _{0 \leq t \leq T}|u(t)|$, and $Y=\{v(t) / v(t) \in C([0, T], \mathbb{R})\}$, with the norm $\|v\|=\max _{0 \leq t \leq T}|v(t)|$.

We define an operator $F: E \rightarrow E$ by

$$
F(u, v)(t)=\left(\begin{array}{c}
F_{1}(u, v)(t) \\
F_{2}(u, v)(t)
\end{array}\right)
$$

where

$$
\begin{aligned}
& F_{1}(u, v)(t)=\int_{0}^{t} G_{\alpha}(t, s) f\left(s, u(s), v(s),{ }^{\rho} \mathcal{D}_{0^{+}}^{\alpha} u(s)\right) d s, \\
& F_{2}(u, v)(t)=\int_{0}^{t} G_{\beta}(t, s) g\left(s, u(s), v(s),{ }^{\rho} \mathcal{D}_{0^{+}}^{\beta} v(s)\right) d s,
\end{aligned}
$$

with

$$
\begin{aligned}
G_{\alpha}(t, s) & =\frac{\rho^{1-\alpha}}{\Gamma(\alpha)} s^{\rho-1}\left(t^{\rho}-s^{\rho}\right)^{\alpha-1}, \\
G_{\beta}(t, s) & =\frac{\rho^{1-\beta}}{\Gamma(\beta)} s^{\rho-1}\left(t^{\rho}-s^{\rho}\right)^{\beta-1} .
\end{aligned}
$$

Theorem 3.4. Assume the hypotheses $(\mathrm{H} 1)-(\mathrm{H} 2)$ hold. If

$$
\frac{T^{\rho \alpha}\left(\lambda_{1}+\lambda_{2}\right)}{\left(1-\lambda_{3}\right) \rho^{\alpha} \Gamma(\alpha+1)}+\frac{T^{\rho \beta}\left(\mu_{1}+\mu_{2}\right)}{\left(1-\mu_{3}\right) \rho^{\beta} \Gamma(\beta+1)}<1 .
$$

Then the problem (1.1)-(1.2) has a unique solution on $[0, T]$.

Proof. By the Lemma 3.3, we will transform the problem (1.1)-(1.2) into a fixed point problem $F(u, v)=(u, v)$, where the operator $F$ is defined by (3.8). Using the Banach contraction principle, we shall show that $F$ has a fixed point. Now for $\left(u_{2}, v_{2}\right),\left(u_{1}, v_{1}\right) \in$ $E$ and $t \in[0, T]$, we get

$$
\begin{aligned}
\left|F_{1}\left(u_{2}, v_{2}\right)(t)-F_{1}\left(u_{1}, v_{1}\right)(t)\right| \leq & \int_{0}^{t} G_{\alpha}(t, s) \mid f\left(s, u_{2}(s), v_{2}(s),{ }^{\rho} \mathcal{D}_{0^{+}}^{\alpha} u_{2}(s)\right) \\
& -f\left(s, u_{1}(s), v_{1}(s),{ }^{\rho} \mathcal{D}_{0^{+}}^{\alpha} u_{1}(s)\right) \mid d s \\
\leq & \int_{0}^{t} G_{\alpha}(t, s)\left|{ }^{\rho} \mathcal{D}_{0^{+}}^{\alpha} u_{2}-{ }^{\rho} \mathcal{D}_{0^{+}}^{\alpha} u_{1}\right| d s .
\end{aligned}
$$

By (H2), we have

$$
\left|{ }^{\rho} \mathcal{D}_{0^{+}}^{\alpha} u_{2}-{ }^{\rho} \mathcal{D}_{0^{+}}^{\alpha} u_{1}\right|=\mid f\left(s, u_{2}(s), v_{2}(s),{ }^{\rho} \mathcal{D}_{0^{+}}^{\alpha} u_{2}(s)\right)
$$




$$
\begin{aligned}
& -f\left(s, u_{1}(s), v_{1}(s),{ }^{\rho} \mathcal{D}_{0^{+}}^{\alpha} u_{1}(s)\right) \mid \\
\leq & \lambda_{1}\left|u_{2}-u_{1}\right|+\lambda_{2}\left|v_{2}-v_{1}\right|+\lambda_{3}\left|{ }^{\rho} \mathcal{D}_{0^{+}}^{\alpha} u_{2}-{ }^{\rho} \mathcal{D}_{0^{+}}^{\alpha} u_{1}\right| .
\end{aligned}
$$

Thus

$$
\left|{ }^{\rho} \mathcal{D}_{0^{+}}^{\alpha} u_{2}-{ }^{\rho} \mathcal{D}_{0^{+}}^{\alpha} u_{1}\right| \leq \frac{1}{1-\lambda_{3}}\left[\lambda_{1}\left|u_{2}-u_{1}\right|+\lambda_{2}\left|v_{2}-v_{1}\right|\right] .
$$

From (3.10), we have

$$
\begin{aligned}
\left|F_{1}\left(u_{2}, v_{2}\right)(t)-F_{1}\left(u_{1}, v_{1}\right)(t)\right| \leq & \frac{1}{1-\lambda_{3}} \int_{0}^{t} G_{\alpha}(t, s)\left[\lambda_{1}\left|u_{2}(s)-u_{1}(s)\right|\right. \\
& \left.+\lambda_{2}\left|v_{2}(s)-v_{1}(s)\right|\right] d s \\
\leq & \frac{T^{\rho \alpha}\left(\lambda_{1}+\lambda_{2}\right)}{\left(1-\lambda_{3}\right) \rho^{\alpha} \Gamma(\alpha+1)} \\
& \times\left(\left\|u_{2}-u_{1}\right\|+\left\|v_{2}-v_{1}\right\|\right) .
\end{aligned}
$$

Then

$$
\left\|F_{1}\left(u_{2}, v_{2}\right)-F_{1}\left(u_{1}, v_{1}\right)\right\| \leq \frac{T^{\rho \alpha}\left(\lambda_{1}+\lambda_{2}\right)}{\left(1-\lambda_{3}\right) \rho^{\alpha} \Gamma(\alpha+1)}\left(\left\|u_{2}-u_{1}\right\|+\left\|v_{2}-v_{1}\right\|\right) .
$$

Similarly, one can find that

$$
\left\|F_{2}\left(u_{2}, v_{2}\right)-F_{2}\left(u_{1}, v_{1}\right)\right\| \leq \frac{T^{\rho \beta}\left(\mu_{1}+\mu_{2}\right)}{\left(1-\mu_{3}\right) \rho^{\beta} \Gamma(\beta+1)}\left(\left\|u_{2}-u_{1}\right\|+\left\|v_{2}-v_{1}\right\|\right) .
$$

Thus it follows from (3.11) and (3.12), that

$$
\begin{aligned}
\left\|F\left(u_{2}, v_{2}\right)-F\left(u_{1}, v_{1}\right)\right\| \leq & {\left[\frac{T^{\rho \alpha}\left(\lambda_{1}+\lambda_{2}\right)}{\left(1-\lambda_{3}\right) \rho^{\alpha} \Gamma(\alpha+1)}+\frac{T^{\rho \beta}\left(\mu_{1}+\mu_{2}\right)}{\left(1-\mu_{3}\right) \rho^{\beta} \Gamma(\beta+1)}\right] } \\
& \times\left(\left\|u_{2}-u_{1}\right\|+\left\|v_{2}-v_{1}\right\|\right) .
\end{aligned}
$$

This implies that by (3.9), $F$ is a contraction operator. As a consequence of Banach's contraction principle, Theorem 2.12, we deduce that $F$ has a unique fixed point which is the unique solution of the problem (1.1)-(1.2) on $[0, T]$. The proof is complete.

Theorem 3.5. Assume that hypotheses $(\mathrm{H} 1)-(\mathrm{H} 3)$ hold. If

$$
\frac{T^{\rho \alpha}}{\rho^{\alpha} \Gamma(\alpha+1)} N_{1}+\frac{T^{\rho \beta}}{\rho^{\beta} \Gamma(\beta+1)} N_{2}<1 .
$$

Then the problem (1.1)-(1.2) has at least one solution on $[0, T]$.

Proof. In the previous theorem, we already transform the problem (1.1)-(1.2) into a fixed point problem $F(u, v)=(u, v)$, where the operator $F$ is defined by (3.8). We demonstrate that $F$ satisfies the assumption of Schauder's fixed point theorem, Theorem 2.13. This could be proved through three steps: 
Step 1. $F$ is a continuous operator. Let $\left(x_{n}, y_{n}\right)_{n \in \mathbb{N}}$ be a real sequence such that $\lim _{n \rightarrow \infty}\left(x_{n}, y_{n}\right)=(x, y)$ in $E$. Then for each $t \in[0, T]$,

$$
\left|F_{1}\left(x_{n}, y_{n}\right)(t)-F_{1}(x, y)(t)\right| \leq \int_{0}^{t} G_{\alpha}(t, s)\left|\begin{array}{c}
f\left(s, x_{n}(s), y_{n}(s),{ }^{\rho} \mathcal{D}_{0^{+}}^{\alpha} x_{n}(s)\right) \\
-f\left(s, x(s), y(s),{ }^{\rho} \mathcal{D}_{0^{+}}^{\alpha} x(s)\right)
\end{array}\right| d s
$$

where

$$
\begin{aligned}
{ }^{\rho} \mathcal{D}_{0^{+}}^{\alpha} x_{n}(t) & =f\left(t, x_{n}(t), y_{n}(t),{ }^{\rho} \mathcal{D}_{0^{+}}^{\alpha} x_{n}(t)\right), \\
{ }^{\rho} \mathcal{D}_{0^{+}}^{\alpha} x(t) & =f\left(t, x(t), y(t),{ }^{\rho} \mathcal{D}_{0^{+}}^{\alpha} x(t)\right) .
\end{aligned}
$$

As a consequence of (H2), we find easily ${ }^{\rho} \mathcal{D}_{0^{+}}^{\alpha} x_{n} \rightarrow{ }^{\rho} \mathcal{D}_{0^{+}}^{\alpha} x$ in $C[0, T]$. In fact we have

$$
\begin{aligned}
\left|{ }^{\rho} \mathcal{D}_{0^{+}}^{\alpha} x_{n}(t)-{ }^{\rho} \mathcal{D}_{0^{+}}^{\alpha} x(t)\right|= & \mid f\left(t, x_{n}(t), y_{n}(t),{ }^{\rho} \mathcal{D}_{0^{+}}^{\alpha} x_{n}(t)\right) \\
& -f\left(t, x(t), y(t),{ }^{\rho} \mathcal{D}_{0^{+}}^{\alpha} x(t)\right) \mid \\
\leq & \lambda_{1}\left|x_{n}(t)-x(t)\right|+\lambda_{2}\left|y_{n}(t)-y(t)\right| \\
& +\lambda_{3}\left|{ }^{\rho} \mathcal{D}_{0^{+}}^{\alpha} x_{n}(t)-{ }^{\rho} \mathcal{D}_{0^{+}}^{\alpha} x(t)\right|
\end{aligned}
$$

Thus

$$
\left|{ }^{\rho} \mathcal{D}_{0^{+}}^{\alpha} x_{n}(t)-{ }^{\rho} \mathcal{D}_{0^{+}}^{\alpha} x(t)\right| \leq \frac{1}{1-\lambda_{3}}\left(\lambda_{1}\left|x_{n}(t)-x(t)\right|+\lambda_{2}\left|y_{n}(t)-y(t)\right|\right) .
$$

Since $\left(x_{n}, y_{n}\right) \rightarrow(x, y)$, then we get ${ }^{\rho} \mathcal{D}_{0^{+}}^{\alpha} x_{n}(t) \rightarrow{ }^{\rho} \mathcal{D}_{0^{+}}^{\alpha} x(t)$ as $n \rightarrow \infty$ for each $t \in[0, T]$. Now let $K_{0}>0$, be such that for each $t \in[0, T]$, we have

$$
\left|{ }^{\rho} \mathcal{D}_{0^{+}}^{\alpha} x_{n}(t)\right| \leq K_{0}, \quad\left|{ }^{\rho} \mathcal{D}_{0^{+}}^{\alpha} x(t)\right| \leq K_{0} .
$$

Then, we have

$$
\begin{aligned}
\left|F_{1}\left(x_{n}, y_{n}\right)(t)-F_{1}(x, y)(t)\right| & \leq \int_{0}^{t} G_{\alpha}(t, s)\left|\begin{array}{c}
f\left(s, x_{n}(s), y_{n}(s),{ }^{\rho} \mathcal{D}_{0^{+}}^{\alpha} x_{n}(s)\right) \\
-f\left(s, x(s), y(s),{ }^{\rho} \mathcal{D}_{0^{+}}^{\alpha} x(s)\right)
\end{array}\right| d s \\
& \leq \int_{0}^{t} G_{\alpha}(t, s)\left|{ }^{\rho} \mathcal{D}_{0^{+}}^{\alpha} x_{n}(s)-{ }^{\rho} \mathcal{D}_{0^{+}}^{\alpha} x(s)\right| d s \\
& \leq \int_{0}^{t} G_{\alpha}(t, s)\left[\left|{ }^{\rho} \mathcal{D}_{0^{+}}^{\alpha} x_{n}(s)\right|+\left|{ }^{\rho} \mathcal{D}_{0^{+}}^{\alpha} x(s)\right|\right] d s \\
& \leq \int_{0}^{t} 2 K_{0} G_{\alpha}(t, s) d s .
\end{aligned}
$$

For each $t \in[0, T]$, the function $s \rightarrow 2 K_{0} G_{\alpha}(t, s)$ is integrable on $[0, t]$, then the Lebesgue dominated convergence theorem imply that

$$
\left|F_{1}\left(x_{n}, y_{n}\right)(t)-F_{1}(x, y)(t)\right| \rightarrow 0 \text { as } n \rightarrow \infty,
$$


and hence

$$
\lim _{n \rightarrow \infty}\left\|F_{1}\left(x_{n}, y_{n}\right)-F_{1}(x, y)\right\|=0 .
$$

Similarly, one can find that

$$
\lim _{n \rightarrow \infty}\left\|F_{2}\left(x_{n}, y_{n}\right)-F_{2}(x, y)\right\|=0 .
$$

Thus it follows from (3.14) and (3.15), that

$$
\lim _{n \rightarrow \infty}\left\|F\left(x_{n}, y_{n}\right)-F(x, y)\right\|=0 .
$$

Consequently, $F$ is continuous.

Step 2. Let

$$
r \geq \frac{\frac{T^{\rho \alpha}}{\rho^{\alpha} \Gamma(\alpha+1)} M_{1}+\frac{T^{\rho \beta}}{\rho^{\beta} \Gamma(\beta+1)} M_{2}}{1-\frac{T^{\rho \alpha}}{\rho^{\alpha} \Gamma(\alpha+1)} N_{1}-\frac{T^{\rho \beta}}{\rho^{\beta} \Gamma(\beta+1)} N_{2}}
$$

We define

$$
P_{r}=\{(u, v) \in E:\|(u, v)\| \leq r\} .
$$

It is clear that $P_{r}$ is a bounded, closed and convex subset of $E$.

Let $(u, v) \in P_{r}$, and $F: P_{r} \rightarrow E$ be the integral operator defined in (3.8), then $F\left(P_{r}\right) \subset P_{r}$. In fact, for each $t \in[0, T]$, we have from $(\mathrm{H} 3)$

$$
\begin{aligned}
\left|{ }^{\rho} \mathcal{D}_{0^{+}}^{\alpha} u(t)\right|=\left|f\left(t, u(t), v(t),{ }^{\rho} \mathcal{D}_{0^{+}}^{\alpha} u(t)\right)\right| \leq & a_{1}(t)+b_{1}(t)|u(t)|+c_{1}(t)|v(t)| \\
& +d_{1}(t)\left|{ }^{\rho} \mathcal{D}_{0^{+}}^{\alpha} u(t)\right|, \\
\left|{ }^{\rho} \mathcal{D}_{0^{+}}^{\beta} v(t)\right|=\left|g\left(t, u(t), v(t),{ }^{\rho} \mathcal{D}_{0^{+}}^{\beta} v(t)\right)\right| \leq & a_{2}(t)+b_{2}(t)|u(t)|+c_{2}(t)|v(t)| \\
& +d_{2}(t)\left|{ }^{\rho} \mathcal{D}_{0^{+}}^{\beta} v(t)\right| .
\end{aligned}
$$

Then

$$
\left|{ }^{\rho} \mathcal{D}_{0^{+}}^{\alpha} u(t)\right| \leq \frac{a_{1}^{*}}{1-d_{1}^{*}}+\frac{b_{1}^{*}+c_{1}^{*}}{1-d_{1}^{*}} r=M_{1}+N_{1} r
$$

and

$$
\left|\rho \mathcal{D}_{0^{+}}^{\beta} v(t)\right| \leq \frac{a_{2}^{*}}{1-d_{2}^{*}}+\frac{b_{2}^{*}+c_{2}^{*}}{1-d_{2}^{*}} r=M_{2}+N_{2} r
$$

Thus

$$
\begin{aligned}
\left|F_{1}(u, v)(t)\right| & \leq \int_{0}^{t} G_{\alpha}(t, s)\left|f\left(s, u(s), v(s),{ }^{\rho} \mathcal{D}_{0^{+}}^{\alpha} u(s)\right)\right| d s \\
& \leq \int_{0}^{t} G_{\alpha}(t, s)\left|{ }^{\rho} \mathcal{D}_{0^{+}}^{\alpha} u(s)\right| d s \\
& \leq \frac{T^{\rho \alpha}}{\rho^{\alpha} \Gamma(\alpha+1)}\left(M_{1}+N_{1} r\right),
\end{aligned}
$$


and

$$
\begin{aligned}
\left|F_{2}(u, v)(t)\right| & \leq \int_{0}^{t} G_{\beta}(t, s)\left|g\left(s, u(s), v(s),{ }^{\rho} \mathcal{D}_{0^{+}}^{\beta} v(s)\right)\right| d s \\
& \leq \int_{0}^{t} G_{\beta}(t, s)\left|{ }^{\rho} \mathcal{D}_{0^{+}}^{\beta} v(s)\right| d s \\
& \leq \frac{T^{\rho \beta}}{\rho^{\beta} \Gamma(\beta+1)}\left(M_{2}+N_{2} r\right) .
\end{aligned}
$$

In consequence, we have

$$
\|F(u, v)\| \leq r
$$

Then $F\left(P_{r}\right) \subset P_{r}$.

Step 3. $F\left(P_{r}\right)$ is relatively compact. Let $t_{1}, t_{2} \in[0, T], t_{1}<t_{2}$ and $(u, v) \in P_{r}$. Then

$$
\begin{aligned}
\left|F_{1}(u, v)\left(t_{2}\right)-F_{1}(u, v)\left(t_{1}\right)\right|= & \mid \int_{0}^{t_{2}} G_{\alpha}\left(t_{2}, s\right) f\left(s, u(s), v(s),{ }^{\rho} \mathcal{D}_{0^{+}}^{\alpha} u(s)\right) d s \\
& -\int_{0}^{t_{1}} G_{\alpha}\left(t_{1}, s\right) f\left(s, u(s), v(s),{ }^{\rho} \mathcal{D}_{0^{+}}^{\alpha} u(s)\right) d s \mid \\
\leq & \int_{0}^{t_{1}} \mid\left[G_{\alpha}\left(t_{2}, s\right)-G_{\alpha}\left(t_{1}, s\right)\right] \\
& \times f\left(s, u(s), v(s),{ }^{\rho} \mathcal{D}_{0^{+}}^{\alpha} u(s)\right) \mid d s \\
& +\int_{t_{1}}^{t_{2}} G_{\alpha}\left(t_{2}, s\right)\left|f\left(s, u(s), v(s),{ }^{\rho} \mathcal{D}_{0^{+}}^{\alpha} u(s)\right)\right| d s \\
\leq & \left(M_{1}+N_{1} r\right)\left[\int_{0}^{t_{1}}\left|\left(G_{\alpha}\left(t_{2}, s\right)-G_{\alpha}\left(t_{1}, s\right)\right)\right| d s\right. \\
& \left.+\int_{t_{1}}^{t_{2}} G\left(t_{2}, s\right) d s\right] .
\end{aligned}
$$

We have

$$
\begin{aligned}
G_{\alpha}\left(t_{2}, s\right)-G_{\alpha}\left(t_{1}, s\right) & =\frac{\rho^{1-\alpha}}{\Gamma(\alpha)} s^{\rho-1}\left[\left(t_{2}^{\rho}-s^{\rho}\right)^{\alpha-1}-\left(t_{1}^{\rho}-s^{\rho}\right)^{\alpha-1}\right] \\
& =\frac{-1}{\alpha \rho^{\alpha} \Gamma(\alpha)} \frac{d}{d s}\left[\left(t_{2}^{\rho}-s^{\rho}\right)^{\alpha}-\left(t_{1}^{\rho}-s^{\rho}\right)^{\alpha}\right]
\end{aligned}
$$

then

$$
\int_{0}^{t_{1}}\left|\left(G_{\alpha}\left(t_{2}, s\right)-G_{\alpha}\left(t_{1}, s\right)\right)\right| d s \leq \frac{1}{\rho^{\alpha} \Gamma(\alpha+1)}\left[\left(t_{2}^{\rho}-t_{1}^{\rho}\right)^{\alpha}+\left(t_{2}^{\rho \alpha}-t_{1}^{\rho \alpha}\right)\right],
$$

we have also

$$
\int_{t_{1}}^{t_{2}} G_{\alpha}\left(t_{2}, s\right) d s=\frac{\rho^{1-\alpha}}{\Gamma(\alpha)} \int_{t_{1}}^{t_{2}} s^{\rho-1}\left(t_{2}^{\rho}-s^{\rho}\right)^{\alpha-1} d s=\frac{-1}{\alpha \rho^{\alpha} \Gamma(\alpha)}\left[\left(t_{2}^{\rho}-s^{\rho}\right)^{\alpha}\right]_{t_{1}}^{t_{2}}
$$




$$
\leq \frac{1}{\rho^{\alpha} \Gamma(\alpha+1)}\left(t_{2}^{\rho}-t_{1}^{\rho}\right)^{\alpha}
$$

Then (3.18) gives

$$
\left|F_{1}(u, v)\left(t_{2}\right)-F_{1}(u, v)\left(t_{1}\right)\right| \leq \frac{M_{1}+N_{1} r}{\rho^{\alpha} \Gamma(\alpha+1)}\left[2\left(t_{2}^{\rho}-t_{1}^{\rho}\right)^{\alpha}+\left(t_{2}^{\rho \alpha}-t_{1}^{\rho \alpha}\right)\right] .
$$

As $t_{1} \rightarrow t_{2}$, the right-hand side of the above inequality tends to zero.

In the same way, we can obtain

$$
\left|F_{2}(u, v)\left(t_{2}\right)-F_{2}(u, v)\left(t_{1}\right)\right| \leq \frac{M_{2}+N_{2} r}{\rho^{\beta} \Gamma(\beta+1)}\left[2\left(t_{2}^{\rho}-t_{1}^{\rho}\right)^{\beta}+\left(t_{2}^{\rho \beta}-t_{1}^{\rho \beta}\right)\right] .
$$

As $t_{1} \rightarrow t_{2}$, the right-hand side of the above inequality tends to zero.

Therefore, the operator $F(u, v)$ is equicontinuous, and by means of the ArzelaAscoli theorem 2.9, we deduce that $F: P_{r} \rightarrow P_{r}$ is continuous, relatively compact and satisfies the assumption of Schauder's fixed point theorem 2.13. Then $F$ has a fixed point which is a solution of the problem (1.1)-(1.2) on $[0, T]$. The proof is complete.

Our next existence result is based on the nonlinear alternative of Leray-Schauder type.

Theorem 3.6. Assume (H1)-(H3) holds. Then the problem (1.1)-(1.2) has at least one solution on $[0, T]$.

Proof. We shall show that the operator $F$ defined in (3.8) satisfies the assumption of Leray-Schauder fixed point theorem 2.14. The proof will be given in several steps.

Step 1. Clearly $F$ is continuous.

Step 2. $F$ maps bounded sets into bounded sets in $E$. Indeed, it is enough to show that for any $\omega>0$, there exist a positive constant $\ell$ such that for each $(u, v) \in B_{\omega}=$ $\{(u, v) \in E:\|(u, v)\| \leq \omega\}$, we have $\|F(u, v)\| \leq \ell$.

For $(u, v) \in B_{\omega}$, we have, for each $t \in[0, T]$

$$
\begin{gathered}
\left|F_{1}(u, v)(t)\right| \leq \int_{0}^{t} G_{\alpha}(t, s)\left|f\left(s, u(s), v(s),{ }^{\rho} \mathcal{D}_{0^{+}}^{\alpha} u(s)\right)\right| d s, \\
\left|F_{2}(u, v)(t)\right| \leq \int_{0}^{t} G_{\beta}(t, s)\left|g\left(s, u(s), v(s),{ }^{\rho} \mathcal{D}_{0^{+}}^{\beta} v(s)\right)\right| d s .
\end{gathered}
$$

By (H3), similarly of (3.16) and (3.17), for each $t \in[0, T]$, we have

$$
\begin{aligned}
\left|F_{1}(u, v)(t)\right| & \leq \frac{T^{\rho \alpha}}{\rho^{\alpha} \Gamma(\alpha+1)}\left(M_{1}+N_{1} \omega\right), \\
\left|F_{2}(u, v)(t)\right| & \leq \frac{T^{\rho \beta}}{\rho^{\beta} \Gamma(\beta+1)}\left(M_{2}+N_{2} \omega\right) .
\end{aligned}
$$


Thus (3.19) and (3.20), implies that

$$
\|F(u, v)\| \leq \frac{T^{\rho \alpha}}{\rho^{\alpha} \Gamma(\alpha+1)}\left(M_{1}+N_{1} \omega\right)+\frac{T^{\rho \beta}}{\rho^{\beta} \Gamma(\beta+1)}\left(M_{2}+N_{2} \omega\right)=\ell .
$$

Step 3. Clearly, $F$ maps bounded sets into equicontinuous sets of $E$. We conclude that $F: E \rightarrow E$ is continuous and completely continuous.

Step 4. A priori bounds. We now show there exists an open set $U \subset E$ with $(u, v) \neq \mu F(u, v)$ for $\mu \in(0,1)$ and $(u, v) \in \partial U$.

Let $(u, v) \in E$ and $(u, v)=\mu F(u, v)$ for some $0<\mu<1$. Thus for each $t \in[0, T]$, we have

$$
\begin{aligned}
& u(t) \leq \mu \int_{0}^{t} G_{\alpha}(t, s)\left|f\left(s, u(s), v(s),{ }^{\rho} \mathcal{D}_{0^{+}}^{\alpha} u(s)\right)\right| d s, \\
& v(t) \leq \mu \int_{0}^{t} G_{\beta}(t, s)\left|g\left(s, u(s), v(s),{ }^{\rho} \mathcal{D}_{0^{+}}^{\beta} v(s)\right)\right| d s .
\end{aligned}
$$

By (H3), for all solutions $(u, v) \in E$ of the problem (1.1)-(1.2), we have

$$
\begin{aligned}
|u(t)| & =\left|\int_{0}^{t} G_{\alpha}(t, s)\right| f\left(s, u(s), v(s),{ }^{\rho} \mathcal{D}_{0^{+}}^{\alpha} u(s)\right)|d s| \\
& \leq \int_{0}^{t} G_{\alpha}(t, s)\left|{ }^{\rho} \mathcal{D}_{0^{+}}^{\alpha} u(s)\right| d s, \\
|v(t)| & =\left|\int_{0}^{t} G_{\beta}(t, s)\right| g\left(s, u(s), v(s),{ }^{\rho} \mathcal{D}_{0^{+}}^{\beta} v(s)\right)|d s| \\
& \leq \int_{0}^{t} G_{\beta}(t, s)\left|{ }^{\rho} \mathcal{D}_{0^{+}}^{\beta} v(s)\right| d s .
\end{aligned}
$$

Then for each $t \in[0, T]$, we have

$$
\begin{aligned}
\left|{ }^{\rho} \mathcal{D}_{0^{+}}^{\alpha} u(t)\right|=\left|f\left(t, u(t), v(t),{ }^{\rho} \mathcal{D}_{0^{+}}^{\alpha} u(t)\right)\right| \leq & a_{1}(t)+b_{1}(t)|u(t)|+c_{1}(t)|v(t)| \\
& +d_{1}(t)\left|{ }^{\rho} \mathcal{D}_{0^{+}}^{\alpha} u(t)\right|, \\
\left|{ }^{\rho} \mathcal{D}_{0^{+}}^{\beta} v(t)\right|=\left|g\left(t, u(t), v(t),{ }^{\rho} \mathcal{D}_{0^{+}}^{\beta} v(t)\right)\right| \leq & a_{2}(t)+b_{2}(t)|u(t)|+c_{2}(t)|v(t)| \\
& +d_{2}(t)\left|{ }^{\rho} \mathcal{D}_{0^{+}}^{\beta} v(t)\right| .
\end{aligned}
$$

Then

$$
\begin{aligned}
\left|{ }^{\rho} \mathcal{D}_{0^{+}}^{\alpha} u(t)\right| & \leq \frac{a_{1}^{*}}{1-d_{1}^{*}}+\frac{b_{1}^{*}}{1-d_{1}^{*}}|u(t)|+\frac{c_{1}^{*}}{1-d_{1}^{*}}|v(t)| \\
& \leq M_{1}+K_{1}|u(t)|+H_{1}|v(t)|
\end{aligned}
$$

and

$$
\left|{ }^{\rho} \mathcal{D}_{0^{+}}^{\beta} v(t)\right| \leq \frac{a_{2}^{*}}{1-d_{2}^{*}}+\frac{b_{2}^{*}}{1-d_{2}^{*}}|u(t)|+\frac{c_{2}^{*}}{1-d_{2}^{*}}|v(t)|
$$




$$
\leq M_{2}+K_{2}|u(t)|+H_{2}|v(t)|
$$

Hence

$$
\begin{aligned}
& |u(t)| \leq \frac{M_{1} T^{\rho \alpha}}{\rho^{\alpha} \Gamma(\alpha+1)}+\int_{0}^{t} G_{\alpha}(t, s)\left(K_{1}|u(s)|+H_{1}|v(s)|\right) d s, \\
& |v(t)| \leq \frac{M_{2} T^{\rho \beta}}{\rho^{\beta} \Gamma(\beta+1)}+\int_{0}^{t} G_{\beta}(t, s)\left(K_{2}|u(s)|+H_{2}|v(s)|\right) d s .
\end{aligned}
$$

Choosing $K_{1}, K_{2}, H_{1}, H_{2} \leq 1$, we find that

$$
\begin{aligned}
|u(t)|+|v(t)| \leq & \frac{M_{1} T^{\rho \alpha}}{\rho^{\alpha} \Gamma(\alpha+1)}+\frac{M_{2} T^{\rho \beta}}{\rho^{\beta} \Gamma(\beta+1)}+\int_{0}^{t}\left(G_{\alpha}(t, s)+G_{\beta}(t, s)\right) \\
& \times(|u(s)|+|v(s)|) d s .
\end{aligned}
$$

After the Gronwall lemma, Lemma 2.11, we have

$$
|u(t)|+|v(t)| \leq\left[\frac{M_{1} T^{\rho \alpha}}{\rho^{\alpha} \Gamma(\alpha+1)}+\frac{M_{2} T^{\rho \beta}}{\rho^{\beta} \Gamma(\beta+1)}\right] \exp \left(\frac{M_{1} T^{\rho \alpha}}{\rho^{\alpha} \Gamma(\alpha+1)}+\frac{M_{2} T^{\rho \beta}}{\rho^{\beta} \Gamma(\beta+1)}\right) .
$$

Thus

$$
\|(u, v)\| \leq\left[\frac{M_{1} T^{\rho \alpha}}{\rho^{\alpha} \Gamma(\alpha+1)}+\frac{M_{2} T^{\rho \beta}}{\rho^{\beta} \Gamma(\beta+1)}\right] \exp \left(\frac{M_{1} T^{\rho \alpha}}{\rho^{\alpha} \Gamma(\alpha+1)}+\frac{M_{2} T^{\rho \beta}}{\rho^{\beta} \Gamma(\beta+1)}\right)=L .
$$

Let

$$
U=\{(u, v) \in E:\|(u, v)\|<L+1\} .
$$

By choosing of $U$, there is no $(u, v) \in \partial U$, such that $(u, v)=\mu F(u, v)$, for $\mu \in(0,1)$. As a consequence of Leray-Schauder's theorem, Theorem 2.14, $F$ has a fixed point $u$ in $U$ which is a solution to (1.1)-(1.2). The proof is finished.

\section{Illustrative Examples}

In this section, we present some examples to illustrate the usefulness of our main results.

Example 4.1. Consider the following coupled system of Katugampola type fractional differential equations

$$
\left\{\begin{array}{l}
{ }^{1} \mathcal{D}_{0^{+}}^{\frac{1}{2}} u(t)=\frac{\arctan (t)}{\left[1+\frac{1}{5}|u(t)|+\frac{1}{50}|v(t)|+\frac{1}{100}\left|{ }^{1} \mathcal{D}_{0^{+}}^{\frac{1}{2}} u(t)\right|\right]} \\
{ }^{1} \mathcal{D}_{0^{+}}^{\frac{1}{3}} v(t)=\frac{1}{10+|u(t)|}+\frac{1}{50+|v(t)|}+\frac{1}{100+t^{2}}\left({ }^{1} \mathcal{D}_{0^{+}}^{\frac{1}{3}} v(t)\right), \\
u(0)=0, v(0)=0
\end{array} \quad t \in[0,1] .\right.
$$


Let

$$
\begin{aligned}
& f\left(t, u, v,{ }^{1} \mathcal{D}_{0^{+}}^{\frac{1}{2}} u\right)=\frac{\arctan (t)}{\left[1+\frac{1}{5}|u(t)|+\frac{1}{50}|v(t)|+\frac{1}{100}\left|{ }^{1} \mathcal{D}_{0^{+}}^{\frac{1}{2}} u(t)\right|\right]} \\
& g\left(t, u, v,{ }^{1} \mathcal{D}_{0^{+}}^{\frac{1}{3}} v\right)=\frac{1}{10+|u(t)|}+\frac{1}{50+|v(t)|}+\frac{{ }^{1} \mathcal{D}_{0^{+}}^{\frac{1}{3}} v(t)}{100+t^{2}}
\end{aligned}
$$

Clearly $f, g:[0,1] \times \mathbb{R}^{3} \rightarrow \mathbb{R}$ are continuous functions and we have

$$
\begin{aligned}
\left|f\left(t, u, v,{ }^{1} \mathcal{D}_{0^{+}}^{\frac{1}{2}} u\right)-f\left(t, \tilde{u}, \tilde{v},{ }^{1} \mathcal{D}_{0^{+}}^{\frac{1}{2}} \tilde{u}\right)\right| \leq & \frac{\pi}{10}|u-\tilde{u}|+\frac{\pi}{100}|v-\tilde{v}| \\
& +\frac{\pi}{200}\left|{ }^{1} \mathcal{D}_{0^{+}}^{\frac{1}{2}} u-{ }^{1} \mathcal{D}_{0^{+}}^{\frac{1}{2}} \tilde{u}\right|, \\
\left|g\left(t, u, v,{ }^{1} \mathcal{D}_{0^{+}}^{\frac{1}{3}} v\right)-g\left(t, \tilde{u}, \tilde{v},{ }^{1} \mathcal{D}_{0^{+}}^{\frac{1}{3}} \tilde{v}\right)\right| \leq & \frac{1}{10}|u-\tilde{u}|+\frac{1}{50}|v-\tilde{v}| \\
& +\frac{1}{100}\left|{ }^{1} \mathcal{D}_{0^{+}}^{\frac{1}{3}} v-{ }^{1} \mathcal{D}_{0^{+}}^{\frac{1}{3}} \tilde{v}\right| .
\end{aligned}
$$

Thus the hypothesis (H2) is satisfied with $\lambda_{1}=\frac{\pi}{10}, \lambda_{2}=\frac{\pi}{100}, \lambda_{3}=\frac{\pi}{200}, \mu_{1}=$ $\frac{1}{10}, \mu_{2}=\frac{1}{50}, \mu_{3}=\frac{1}{100}$ and

$$
\frac{T^{\rho \alpha}\left(\lambda_{1}+\lambda_{2}\right)}{\left(1-\lambda_{3}\right) \rho^{\alpha} \Gamma(\alpha+1)}+\frac{T^{\rho \beta}\left(\mu_{1}+\mu_{2}\right)}{\left(1-\mu_{3}\right) \rho^{\beta} \Gamma(\beta+1)} \simeq 0.5318<1 .
$$

Therefore, (3.9) is satisfied. Hence, all conditions of theorem 3.4 hold. thus the coupled system (4.1) has a unique solution on $[0,1]$.

Example 4.2. Consider the following coupled system of Katugampola type fractional differential equations

$$
\left\{\begin{array}{l}
{ }^{1} \mathcal{D}_{0^{+}}^{\frac{3}{4}} u(t)=\frac{1+u(t)+v(t)}{\sqrt{16+t^{2}}}+\frac{\arctan (t)}{2}\left({ }^{1} \mathcal{D}_{0^{+}}^{\frac{3}{4}} u(t)\right) \\
{ }^{1} \mathcal{D}_{0^{+}}^{\frac{4}{5}} v(t)=\frac{1+(\sqrt[3]{|u(t)|}+\sqrt[3]{|v(t)|})^{3}}{e^{t^{2}}}+\frac{\pi \ln \left(\left|{ }^{1} \mathcal{D}_{0^{+}}^{\frac{4}{5}} v(t)\right|\right)}{8+t^{2}}, t \in[0,1] . \\
u(0)=0, v(0)=0,
\end{array}\right.
$$

Let

$$
\begin{aligned}
& f\left(t, u, v,{ }^{1} \mathcal{D}_{0^{+}}^{\frac{3}{4}} u\right)=\frac{1+u(t)+v(t)}{\sqrt{16+t^{2}}}+\frac{\arctan (t)}{2}\left({ }^{1} \mathcal{D}_{0^{+}}^{\frac{3}{4}} u(t)\right) \\
& g\left(t, u, v,{ }^{1} \mathcal{D}_{0^{+}}^{\frac{4}{5}} v\right)=\frac{1+(\sqrt[3]{|u(t)|}+\sqrt[3]{|v(t)|})^{3}}{e^{t^{2}}}+\frac{\pi \ln \left(\left|{ }^{1} \mathcal{D}_{0^{+}}^{\frac{4}{5}} v(t)\right|\right)}{8+t^{2}} .
\end{aligned}
$$


Clearly $f, g:[0,1] \times \mathbb{R}^{3} \rightarrow \mathbb{R}$ are continuous functions and we have

$$
\begin{aligned}
\left|f\left(t, u, v,{ }^{1} \mathcal{D}_{0^{+}}^{\frac{3}{4}} u\right)\right|= & \frac{1}{\sqrt{16+t^{2}}}+\frac{1}{\sqrt{16+t^{2}}}|u(t)|+\frac{1}{\sqrt{16+t^{2}}}|v(t)| \\
& +\frac{\arctan (t)}{2}\left|{ }^{1} \mathcal{D}_{0^{+}}^{\frac{3}{4}} u\right| \\
\left|g\left(t, u, v,{ }^{1} \mathcal{D}_{0^{+}}^{\frac{4}{5}} v\right)\right|= & \frac{1}{4} e^{-t^{2}}+\frac{1}{4} e^{-t^{2}}|u(t)|+\frac{1}{4} e^{-t^{2}}|v(t)|+\frac{\pi}{8+t^{2}}\left|{ }^{1} \mathcal{D}_{0^{+}}^{\frac{4}{5}} v(t)\right| .
\end{aligned}
$$

Thus the hypothesis (H3) is satisfied with

$$
\begin{array}{ll}
a_{1}(t)=b_{1}(t)=c_{1}(t)=\frac{1}{\sqrt{16+t^{2}}}, & d_{1}(t)=\frac{\arctan (t)}{2}, \\
a_{2}(t)=b_{2}(t)=c_{2}(t)=\frac{1}{4} e^{-t^{2}}, & d_{2}(t)=\frac{\pi}{8+t^{2}}, \\
a_{1}^{*}=b_{1}^{*}=c_{1}^{*}=a_{2}^{*}=b_{2}^{*}=c_{2}^{*}=\frac{1}{4}, & d_{1}^{*}=d_{2}^{*}=\frac{\pi}{8}<1, \\
M_{1}=M_{2}=\frac{2}{8-\pi}, & N_{1}=N_{2}=\frac{4}{8-\pi},
\end{array}
$$

and the condition (3.13),

$$
\frac{T^{\rho \alpha}}{\rho^{\alpha} \Gamma(\alpha+1)} N_{1}+\frac{T^{\rho \beta}}{\rho^{\beta} \Gamma(\beta+1)} N_{2} \bumpeq 0.8753<1 .
$$

It follows from Theorem 3.5 and Theorem 3.6, that the problem (4.2) has at least one solution on $[0,1]$.

\section{Conclusion}

In this paper, the existence and uniqueness of solution for a new coupled system of nonlinear fractional differential equations involving Katugampola fractional derivative in bounded domain have been discussed. For our discussion, we have used the Banach contraction principle, Schauder, nonlinear alternative Leray-Schauder fixed point theorems. From the above discussion, it is expected that this may provide a new direction to the study of coupled systems of nonlinear fractional differential equations.

\section{References}

[1] B. Ahmad and J.J. Nieto. Existence results for a coupled system of nonlinear fractional differential equations with three point boundary conditions. Comput. Math. Appl., 58:1838-1843, 2009. 
[2] B. Ahmad and S.K. Ntouyas. Existence results for a coupled system of Caputo type sequential fractional differential equations with nonlocal integral boundary conditions. Appl. Math. Comput., 266:615-622, 2015.

[3] B. Ahmad, S.K. Ntouyas, and A. Alsaedi. On a coupled system of fractional differential equations with coupled nonlocal and integral boundary conditions. Chaos Solitons Fractals., 83:234-241, 2016.

[4] K. Diethelm. The Analysis of Fractional Differential Equations. Springer, Berlin, 2010.

[5] A. Granas and J. Dugundji. Fixed Point Theory. Springer-Verlag, New York, 2003.

[6] T.H. Gronwall. Note on the derivatives with respect to a parameter of the solutions of a system of differential equations. Ann. Math., 20:292-296, 1918-1919.

[7] N. Jin and S. Sun. On a coupled system of fractional compartmental models for a biological system. Adv. Difference Equ., 146, 2017.

[8] U.N. Katugampola. New approach to a generalized fractional integral. Appl. Math. Comput., 218(3):860-865, 2011.

[9] U.N. Katugampola. A new approach to generalized fractional derivativesl integral. Bull. Math. Anal. Appl., 6(4):1-15, 2014.

[10] A.A. Kilbas, H.H. Srivastava, and J.J. Trujillo. Theory and Applications of Fractional Differential Equations. Elsevier Science B.V, Amsterdam, 2006.

[11] K.S. Miller, B. Ross, and J.J. Trujillo. An Introduction to the Fractional Calculus and Fractional Differential Equations. Wiley, New York, 1993.

[12] I. Podlubny. Fractional Differential Equations, Mathematics in Science and Engineering. Academic Press, New York, 1999.

[13] M. Rehman and R.A. Khan. A note on boundary value problems for a coupled system of fractional differential equations. Compu. Math. Appl., 61:2630-2637, 2011.

[14] S.G. Samko, A.A. Kilbas, and O.I. Marichev. Fractional Integral and Derivatives (Theory and Applications). Gordon and Breach, Switzerland, 1993.

[15] X. Su. Boundary value problem for a coupled system of nonlinear fractional differential equations. Compu. Math. Appl., 22:64-69, 2009.

[16] X. Su, Q. Li, and Y. Li. Existence and uniqueness of solutions for a coupled system of multi-term nonlinear fractional differential equations. Compu. Math. Appl., 64:3310-3320, 2012. 
[17] C. Zhai and R. Jiang. Unique solutions for a new coupled system of fractional differential equations. Adv. Difference Equ., 1, 2018. 